\title{
Mass Spectrometry and Metabolomics-New Approaches for Helminth Biochemical Studies
}

\author{
Estela O. Lima, Cibele Z. Esteves, Diogo N. Oliveira, \\ Tatiane M. Guerreiro, Carlos F. O. R. Melo and \\ Rodrigo R. Catharino
}

Additional information is available at the end of the chapter

http://dx.doi.org/10.5772/65040

\begin{abstract}
Metabolomics, the study of the endogenously synthesized small molecules repertoire (nonproteinaceous), is of great relevance for establishing a wide view of cell physiology at specific moments, linking metabolic profiles to phenotypes and genotypes. To better understand biological systems, such as helminths life cycle, helminthic infection, and host-parasite interaction, metabolomics studies are crucial. For that, mass spectrometrybased metabolomics is the most popular strategy. Nontargeted metabolomics allows researchers to profile entire metabolomes present in cells, tissues, biofluids, or even samples as complex as stools. Through different mass spectrometric techniques, it is possible to unveil chemical markers for helminths, such as Schistosoma mansoni (a trematode) and Ascaris lumbricoides (a nematode), in addition to study mechanisms of action for different drugs, which targets parasites. Therefore, mass spectrometry allows designing biochemical pathways that may clarify the processes of parasite life cycle, helminthic infection, and host-parasite interaction, providing targets to further interference for parasite control or even infection treatment.
\end{abstract}

Keywords: metabolomics, mass spectrometry, helminths, Ascaris lumbricoides , Schistosoma mansoni 


\section{Introduction}

\subsection{Metabolomics and mass spectrometry in biomedical research}

The composition of the intracellular environment depends on harmonic and orchestrated synthesis and interaction of molecules that are essential for cellular survival and healthy life maintenance [1], and it is true for both eukaryotic and prokaryotic organisms. Eukaryotic cells present a higher degree of intricacy compared with prokaryotic cells because of the huge biochemical complexity they present [2]. Helminths represent a pluricellular eukaryotic group of worms that establish parasitic relationships with humans, which has been registered since ancient Greek writings [3]. Structurally, helminths are completely different from Homo sapiens, and it is assured that peculiar metabolic differences exist between these organisms. These differences enable understanding and studying helminth-human interaction, transmission routes, and life cycles through molecular studies, the cornerstone in all fields of biomedical research.

The search for faster, more accurate methodologies that describe the biochemical/pathophysiological status of an organism of interest has driven major efforts in biomedical research in the late years in order to meet the clinical needs for a health community [4]. Bioanalytical methodologies have then emerged as suitable choices for expeditious approaches that exhibit great resolution and sensitivity, thus generating the development of more accurate and precise methodologies. In instrumental bioanalysis, several compounds of interest can be measured and/or monitored depending on their molecular nature. As there is no "one-size-fits-all" approach, different molecular classes have demanded the creation of different analytical strategies in which both comprehensive and specific analyses can be performed. This, therefore, has elicited the development of the "omics" strategies, which were subdivided according to the needs of the researchers. For example, for large and bulky molecules such as proteins, a wide array of analytical methods compose what is called the proteomics approach, which is dedicated to study each and every aspect of proteins and their levels, interactions, and structure in biological systems [5]. The same rationale was then applied for other biological components, such as genes (genomics) [6], lipids (lipidomics) [7], and small molecules/ metabolites (metabolomics) [8].

Metabolomics can be understood in more detail as the systematic study of small, nonprotein, both endogenous and exogenous molecules, which represent the end of the entire biological chain started by gene expression (i.e., phenotype) [9]. Metabolomics studies usually measure compound classes such as lipids, nucleic acids, amino acids, and glycans, composing a useful biochemical platform for studying mechanisms of interactions, signalling synthetic pathways of systems and molecules under different metabolic conditions (e.g., diseases), providing specific molecules or molecular groups that characterize such conditions: the biomarkers. This assists in understanding, diagnosing, preventing, and predicting conditions, as well as helps assessing risks and benefits of pharmacological interventions [10].

Cell metabolism is one of the challenges that have been the driving force of metabolomics over the years [11]. The efforts on development were to obtain increasingly accurate and well- 
resolved methods, introducing more sophisticated and specific methods such as nuclear magnetic resonance (NMR) and mass spectrometry (MS) [12]. This made room for a number of improvements also in data processing and bioinformatics, where multivariate analyses and big data analytics found a whole new application in resolving and integrating complex biological matrices and systems [13], giving rise to the four main metabolomics strategies: metabolic profiling, the identification and quantification of known metabolites that are related to a specific number of metabolic pathways [14]; metabolic fingerprinting, which comprises fast-paced analysis to classify a determined sample during high-throughput screenings (positive/negative or disease/health) [15]; metabolic footprint, which analyses the extracellular metabolites (i.e., excretion/secretion products) of a given organism [16]; and targeted analysis, a series of quali-quantitative analyses of any given known metabolite in an orga-nism or a metabolic pathway [17].

The technical aspects of both NMR and MS place them both as the main tools for metabolomics studies, especially for their complementary nature. NMR is a spectroscopy-based technique that relies on the incidence of a magnetic field over the nuclei of atoms with nonzero spin counts, such as ${ }^{1} \mathrm{H},{ }^{13} \mathrm{C}$, and ${ }^{15} \mathrm{~N}$, thus providing accurate structural and quantitative information of the metabolic profile under study [18]. There are several advantages associated with the use of NMR as an analytical tool for metabolomics; as it is not a destructive analysis, i.e., it is possible to recover the sample after the analysis, which is useful especially for target analyses. Moreover, NMR requires little sample preparation and shows great reproducibility, two desirable features for bioanalyses [19]. In contrast, some disadvantages hamper the wide distribution of NMR as a routine approach; the high costs associated with instrument maintenance and the need for relatively high amounts of the analyte usually turn down NMR as a high-throughput choice, which finds its best use as a research tool.

Mass spectrometry, on the other hand, despite being sample-destructive, shows great potential for routine analyses. MS-based bioanalytical systems show superior performance in detection and quantification limits, easily reaching a working range of nano to picomoles [20]. Relying on a basic structure of ionization source-mass analyser, MS brings a wide array of combinations of both, which comprehends virtually any type of sample for metabolomics studies [21], in addition to the possibility of coupling with chromatographic approaches such as gas chromatography (GC) and high-performance liquid chromatography (HPLC) [22]. Moreover, structural elucidation strategies with MS are diverse and can be suited according to the needs and to the instrument available, either with mass accuracy on high-resolution instruments or with fragmentation profile (MS/MS) in standard devices-or even a combination of the two [23]. These factors contributed to the popularization of MS among researchers and clinical experts and have, therefore, provided the basis for great development in this area.

Hence, this chapter will provide a comprehensive overview on the application of metabolomics analysis and strategies developed for the analyses of helminths, with a particular focus on MS, reinforcing the great importance of these organisms for general public health. The main aim is to address recent analytical chemistry methodologies, challenges to overcome and promising results, expanding possibilities at helminthiasis research, and thus contributing with advances in the area. 


\section{Biochemical characterization of helminthic parasites}

\subsection{Schistosoma mansoni biochemical characterization}

S. mansoni metabolomics studies are conducted to improve the knowledge of disease pathology, to evaluate the behaviour and infectivity of different strains, to elucidate mechanisms of action, and to evaluate the efficacy of available drugs in addiction to discover biomarkers that can be applied as diagnostic and drug targets [24].

The main analytical metabolomics approaches are MS and NMR [25], and both of them allow performing target or untargeted analysis, which are mostly focused on two classes of metabo-lites: lipids and oligosaccharides. In case of living organisms, such as parasites, lipids are necessary for cell-cell signalling, for protection from host immunity, for maintenance of integrity and parasite structure during the life cycle and are also involved in the mechanisms of sexual development, egg production, and cercaria penetration [26-28]. Concerning oligosaccharides, these molecules are precursors of glycoconjugates such as glycoproteins and glycolipids that are mainly involved in development of innate and adaptive immune response in the host, parasite survival, and the chronic infection establishment [29, 30]. Lipids as well glycans may change as long as the parasite develops, thus it is crucial to understand metabolomics patterns according to the different lifecycle stages for each parasite studied.

\subsubsection{Metabolomics approach for S. mansoni lifecycle stages}

\subsubsection{Mature and immature eggs}

The main class of lipids present at S. mansoni eggs is glycosphingolipids [31], which are involved in interactions with the different affected organs, such as gut, lungs, or liver, facilitating its passage through the tissues [32]. Glycosphingolipids are also reported to be associated with egg's high immunogenicity and pathogenicity in different strains [33]. For example, the granuloma formation occurs by the reaction of host immune response mediated by parasite glycoconjugates; therefore, the greater abundance of glucosylceramides (GlcCer) in one strain compared to another, the higher pathogenicity $[32,34]$. The same study using high-resolution mass spectrometry (HRMS) showed that phosphatidic acid (PA), phosphatidylserine (PS), phosphatidylethanolamine (PE), triacylglycerol (TG), and diacylglycerol (DG) are involved in the differentiation of $S$. mansoni strains [34]. In respect to maturation, immature and mature eggs present also been differentiated using Matrix-Assisted Laser Desorption/ Ionization-Time of Flight Mass Spectrometry (MALDI-TOF-MS). This research showed that immature eggs have a common $\mathrm{N}$-glycan profile and are formed predominantly of nonspecific oligomanoses, while mature eggs have highly complex and fucosylated N-glycans [30]. Therefore, lipid molecules in eggs show differences according to maturation phases and diverge between distinct strains. 


\subsubsection{Miracidium}

This is about a free-living and motile form that penetrates in Biomphalaria sp. snails, the intermediate host of the $S$. masoni lifecycle. Different strains were analyzed by HRMS, and the results showed the possibility to differentiate two strains of miracidium by identifying different lipids: triacylglycerol (TG), phosphatidylserine (PS), phosphatidic acid (PA), phosphatidylinositol-ceramide (PI-Cer), and dodecanoid acid (DA). The most interesting lipid among them is the last compound, reported to be responsible for facilitating the penetration of miracidium in snails [27]. Considering glycolipid studies through MALDI-TOF-MS analysis, miracidium glycolipids have been almost devoid of LeX- or LN-motifs, what is coherent with previous data that showed snail infection is apparently independent of LeX-motifs, but LDNmotifs are fairly present in miracidium and snail. Observing that the parasite presented the same spectra compared to the intermediate host, miracidium appear to develop a snailcompatible glycosylation pattern [30]. These molecular studies show lipid variability and/or similarity among different lifecycle stages, opening opportunities to better understand interactions between parasite and host/intermediate host or to interfere at metabolic pathways, as long as we know where and how [30,35].

\subsubsection{Cercaria}

Cercaria, the final larval stage of $S$. mansoni lifecycle, produces secretions that facilitate skin penetration. These fluids are reported to be weak immunogenic and composed of glucoproteins and glycolipids. Mass spectrometry approaches demonstrate the predominance of ceramides-linked glucose and/or galactose with fucose and xylose cores at cercaria secretions $[30,33,36]$. Another study also determined the cercaria differentiation between two S. mansoni strains by HRMS. The lipids TG, DG, PE, PA, and phosphatidylglycerol (PG) were reported to be responsible for this discrimination [27].

\subsubsection{Adults}

The tegument surface structure of S. mansoni adult worms is crucial for parasite survival and modulation of host immune response. This structure is syncytial and is covered by two closely apposed lipid bilayers, one of them is an inner plasma membrane and the other an outer membranocalyx complex [28, 37]. Mass spectrometry studies conducted with tegument and whole worm demonstrate an enrichment of the phosphatidylcholine (PC) and PS of tegumental compositions, but this phenomena does not occur with PE and PI, which presents the same composition for the two worm fractions. The tegumental membranes are also enriched with lysophospholipids, mainly lyso-PS and lyso-PE containing eicosanoic acid (20:1) [28].

As regards glycans, their composition dramatically changes during the development stages. Comparing with early life-stages, adult worms present smaller ratio between complex typeglycans and oligomannosidic glycans and the antigenic core composed by xylose is undetectable [30].

Mass spectrometry imaging (MSI) enables the study of spatial distribution of biomarkers. Ferreira and co-workers (2014) used MALDI-MSI to image two different S. mansoni strains. 
Figure 1 shows the sum of all ions obtained by the imaging analysis where it is possible to detect both male and female worms' bodies. The spectra obtained from the single worms were compared with the pair and enabled the election of specific ions for male and female worms [34].

A
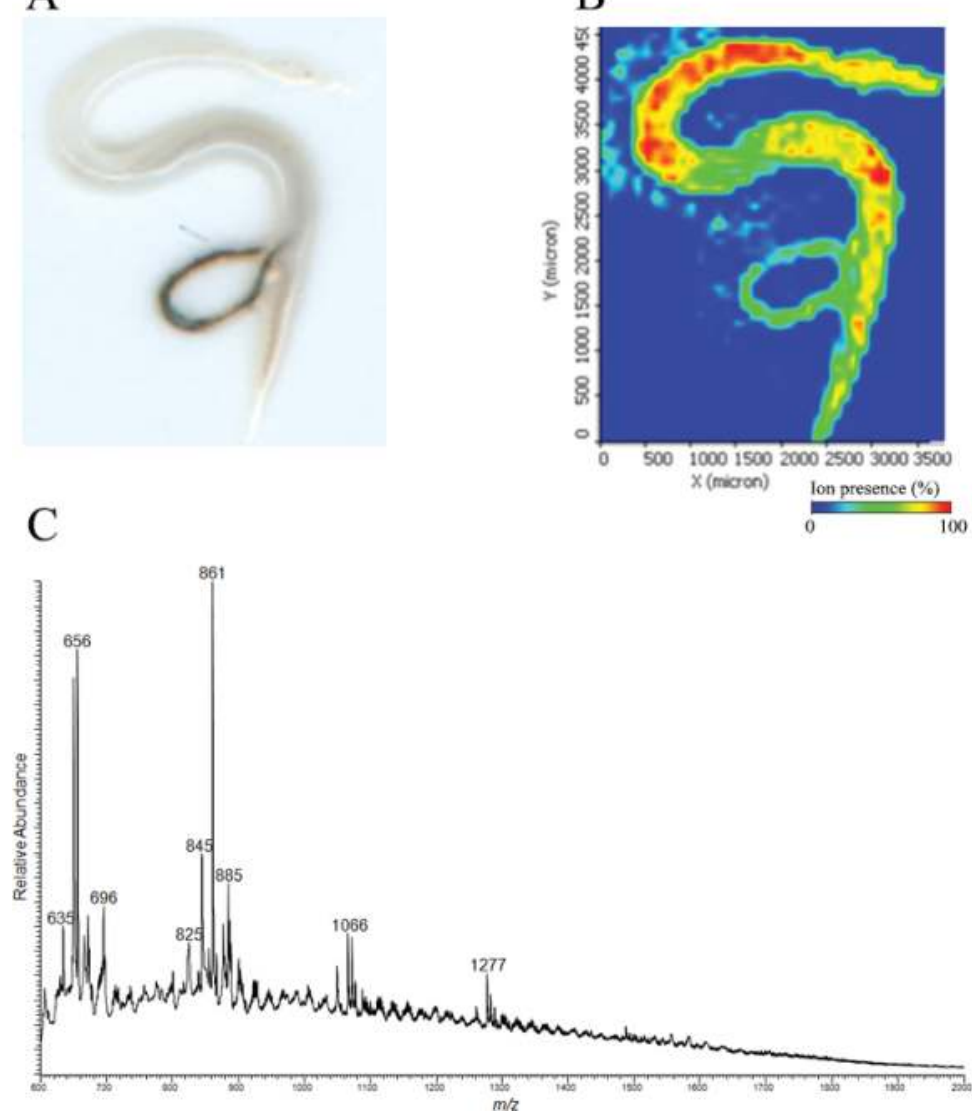

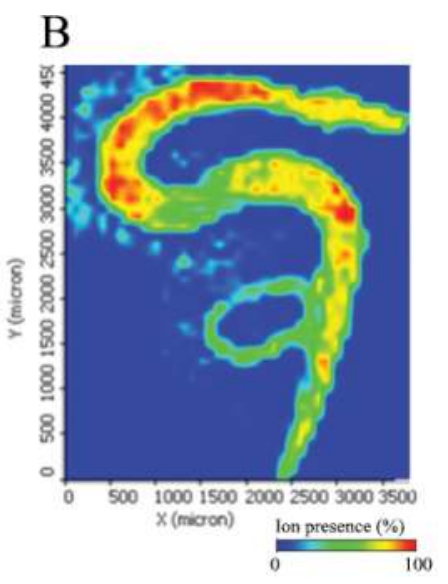

Figure 1. Representative fingerprinting of Schistosoma mansoni adult couple worms. The imaging represents a sum of all ions within mass range 600-2000 m/z. (A) Picture of worms analyzed. Gynaecophoric channel in male's body (clear) holding female's body (dark). (B) Worms' image generated by MALDI-MSI instrument. Illustrative picture of fingerprinting analysis. (C) Representative fingerprinting (total ion current) spectra. Positive ion mode.

The same study determined the ions responsible for the differentiation of male and female worms of two different strains and their localization in a worm body. The fingerprint of each worm was submitted to Principal Component Analysis (PCA), and the groups of strains are discriminated, enabling the selection of chemical markers for each group. New MS/MS imaging was performed and the distribution of the parental ions selected by PCA can be seen in Figures 2 and 3. 
Figure 2 shows the male and female image of $\mathrm{BH}$ strain obtained by MALDI-MSI analysis. Female worm presents the predominance of PC (823, 665 and $761 \mathrm{~m} / \mathrm{z})$ and PG $(764 \mathrm{~m} / \mathrm{z})$ distributed to reproductive and digestive systems. Male worm presents diacylcglycerol (DAG) (635 and $651 \mathrm{~m} / \mathrm{z}$ ) in ventral sucker and gynaecophoric channel, triacylglycerol (TAG) (846 and $886 \mathrm{~m} / \mathrm{z})$ in ventral sucker and reproductive system, phosphatidic acid (PA) $(675 \mathrm{~m} / \mathrm{z})$ in ventral sucker and gynaecophoric channel, PI $(862 \mathrm{~m} / \mathrm{z})$ in reproductive and digestive systems and ventral sucker, and finally, PC $(887 \mathrm{~m} / \mathrm{z})$ in reproductive system and ventral sucker.

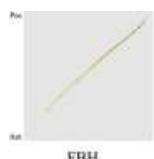

$\mathrm{FBH}$
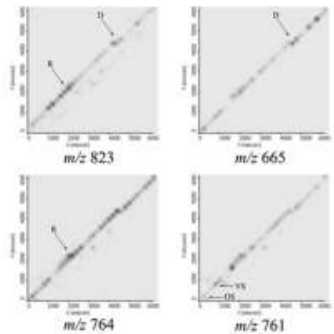
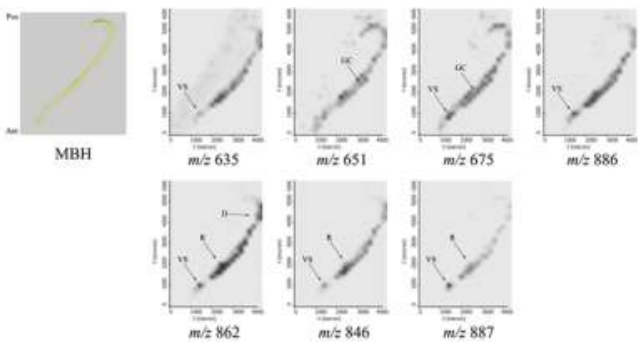
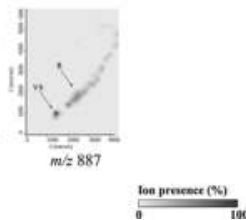

Figure 2. Adult worms generated by MS, selected using each of the parent ions in MS/MS mode. Images were acquired as one run for each $\mathrm{m} / \mathrm{z}$. Female and male $\mathrm{BH}$ strain ( $\mathrm{FBH}$ and $\mathrm{MBH}$, respectively). Pos, posterior portion; Ant, anterior portion; R, reproductive system; D, digestive system; VS, ventral sucker; OS, oral sucker; and GC, gynaecophoric channel.
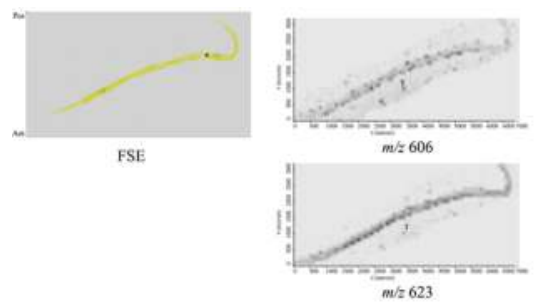

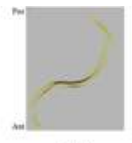

MSE
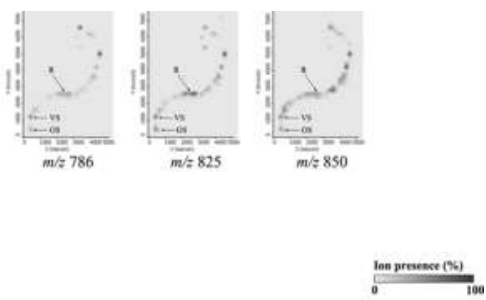

Figure 3. Adult worm images generated by MS, selected using each of the parent ions in MS/MS mode. Images were acquired as one run for each $\mathrm{m} / \mathrm{z}$. Female and male SE strain (FSE and MSE, respectively). Pos, posterior portion; Ant, anterior portion; R, reproductive system; VS, ventral sucker; and OS, oral sucker.

Figure 3 shows male and female image of SE strain obtained by MALDI-MSI analysis. Female worm presents the predominance of PI $(623 \mathrm{~m} / \mathrm{z})$ and DAG $(606 \mathrm{~m} / \mathrm{z})$ distributed mainly in tegument while male worm presents TAG $(786$ and $850 \mathrm{~m} / \mathrm{z})$ and PC $(825 \mathrm{~m} / \mathrm{z})$ in ventral and oral suckers and reproductive system.

Ferreira and coworkers (2014) demonstrate that it is possible to determine spatial distribution of biomarkers for parasites and also show that more studies are necessary to understand the function of each chemical markers according to the organs they are located, searching for its influence through pathogenicity of different strains. Furthermore, a new platform of study 
putting together metabolomic and mass spectrometry techniques was proposed and was named as Parasitomics.

\subsection{Ascaris lumbricoides and chemical markers at moulting process}

According to Pullan and coworkers [38] and CDC [39], it is estimated that 800-1200 million people are infected with Ascaris lumbricoides worldwide and more than 60,000 deaths occur annually because of Ascariasis (spectrum of disease caused by Ascaris lumbricoides infection). World Health Organization has applied some strategies for disease burden control, such as deworming programmes [40]. In case of soil-transmitted helminthiasis, the main public health strategy is the mass drug administration (MDA), which consists of anthelmintic drug treatment regularly provided to risk population, mainly school-aged children [40,41]. Despite the great efforts, approximately $30 \%$ of children are really receiving the anthelmintic treatment in the whole world [42]. Besides the absence of total coverage of MDA worldwide, there is still another issue to pay attention, the development of anthelmintic drug resistance [43-45].

Taking into account all of these issues cited above, beyond the disability-adjusted life years (DALYs) in consequence of morbidity and mortality indexes, it is necessary to better comprehend and to deal with helminthic parasites, such as Ascaris lumbricoides, in a different point of view. It is urgent to evaluate and discover different and better ways to control and eliminate it. Therefore, to outline strategies that are suitable for its life cycle could bring light to management of helminthiasis. Since part of the development of $A$. lumbricoides happens in the soil $[46,47]$, it is therefore reasonable to consider that breaking the life cycle during the phases in the environment would be a valuable attempt in containing the infection in endemic areas [48].

Molecular detailed analysis of the diverse A. lumbricoides forms may elucidate new targets for parasite control. Metabolic fingerprinting using mass spectrometry has been widely employed, targeting parasite-related metabolites [34, 49,50]. The trend of establishing target molecules and linking them with important metabolic pathways in the parasite life cycle broadens the knowledge in the field, generating a platform that will assist in better knowledge on their biology, elucidation of survival mechanisms, pharmacodynamics, diagnostic methodologies, development of pharmaceutical products to eradicate these diseases, whether in soil or living hosts, etc. [50, 51].

Melo and coworkers aimed to characterize, in a simple way, the larval development stages of A. lumbricoides (the traditional human ascariasis agent) by metabolic fingerprinting using HRMS from stool samples [52]. The molecular elucidation in different life stages presents a huge potential for application in parasite infection control, breaking the life cycle and reducing infection and re-infection of exposed human beings, mainly at endemic zones.

From Ascaris lumbricoides specimens, it is possible to separate different development larval stages using optical microscopy. Melo's researchers group analyzed stool samples collected from school-aged children at Brazil. Nonembryonated and embryonated eggs, containing L1 and L3 larvae, could be separated from stool pool. L2 stage larvae were excluded from samples because it is an overlapping period between the end of L1 and the beginning of L3, what makes it difficult to identify and isolate. Considering the high sensitivity of mass spectrometer 
analyser, it was necessary to clean as much as possible this complex sample, what favors the real identification of significant molecules [52].

For molecular screening like this, starting from a complex sample, it is necessary to do a multivariate statistical analysis for correct identification of targets. For the present study, Principal Component Analysis (PCA) was chosen to statistically determine relevant groups of ions, what led to identification of the correspondent molecular biomarkers for each development stage under analysis. From the elected ions by PCA, it was possible to achieve the most suitable chemical markers through metabolome databases analysis, along with a good literature review. ESI-HRMS analysis of Ascaris lumbricoides specimens showed different spectral signals among the egg, L1 and L3, and PCA statistical analysis elected distinct chemical markers for each structure/larval stage.

\subsubsection{Metabolomics approach for A. lumbricoides lifecycle stages}

\subsubsection{Eggs}

Considering eggs analysis, it was possible to identify five biomarkers: hexadecenal $(515 \mathrm{~m} / \mathrm{z})$, 21-Methyl-8Z-pentatriacontene (522 m/z), 3,7,11,15-Tetramethyl tetratriacontane $(565 \mathrm{~m} / \mathrm{z})$, and two unsaturated fatty acids, corresponding to $537 \mathrm{~m} / \mathrm{z}$. The phospholipid hexadecenal may probably be a by-product of sphingosine-1-phosphate (S1P) degradation. Hexadecenal is associated with cytoskeleton reorganization, JNK-dependent apoptosis, and proliferation signalling pathways. Since the analyzed eggs were under embryogenesis, JNK pathway may favor cell proliferation and embryo development [53-55]. Taking into account that hexadecenal appears only in the mass spectra of eggs, it may be acting in signalling during embryonic development process according to weather conditions, food availability and population, among others [56].

Two other signals elected by PCA, 21-Methyl-8Z-pentatriacontene (522 m/z) and 3,7,11,15Tetramethyl Tetratriacontane $(565 \mathrm{~m} / \mathrm{z})$, correspond to signalling molecules in insects, although their function is not clear yet. There were still two unsaturated fatty acids elected for eggs $(537 \mathrm{~m} / \mathrm{z})$ that, based at their carbon chain, it is supposed they have a role at preventing water loss, considering that fertilized eggs are constantly at high risk of dehydration in the soil $[57,58]$.

\subsubsection{L1 moulting stage}

Considering biomarkers statistically elected for L1, a total of four molecules were selected. One of them, 2-deoxyecdysone-22-phosphate $(563 \mathrm{~m} / \mathrm{z})$, is a steroid hormone source found during insects embryogenesis. These steroid hormones, commonly called ecdysones, are associated with control of development in insects and nematodes, processes called moulting [59]. Taking into account that ecdysone synthesis depends on cholesterol [60], it is necessary for the nematode to absorb it. Therefore, it could explain the election of cholesteryl ester (CE) as a second biomarker at L1 stage [61, 62]. 
The lipid composition may vary according to environmental conditions and parasite life stage. Elected as L1 biomarker, cardiolipins are examples of molecules present at the inner mitochondrial membranes [63], whose constitution may vary to adapt to different aeration conditions. Considering that the development of $A$. lumbricoides occurs through different life stages with distinct air availability [64], it is coherent that oxidative stress conditions interfere with mitochondrial lipid composition. In addition to cardiolipins, sphingolipids (SPL) were one of the chemical markers elected at L1 stage. SPL is an important class of lipids and may be modified according to the cycle life stage, just similarly as it happens with insects' development [65].

\subsubsection{L3 moulting stage}

The last larval stage studied at Melo's work was L3, and one of the possible markers was Dimethylheptatriacontane. It is a signalling molecule involved with sexual communication between arthropods, better known as pheromone [66]. Considering nematodes and arthropods are closely related, some hypothesis were raised about its function at A. lumbricoides L3: (i) it could play the same role as in arthropods; (ii) to stimulate sexual differentiation during parasite development [67]; (iii) to act as a "messenger" during development according to environmental conditions [56]. Depending on the life stage, this signalling molecule may present more than one function, but the exact role of dimethylheptatriacontane at nematodes is still to be unveiled.

Considering all biomarkers elected for different lifecycle stages for Ascaris lumbricoides, it is possible to observe that there is a whole metabolic parasite world to discover and establish connections. Therefore, it is too important to keep looking for new biochemical pathways that explain parasite similarities, survival, development, host invasiveness, and immunogenicity. Only the entire knowledge will answer the unsolved questions and allow human interference to improve health conditions and social development.

\section{Searching for drug targets at helminthiasis-Praziquantel and Schistosoma mansoni chemical targets}

It is estimated that about 2 billion people in the world, especially the inhabitants of developing regions such as sub-Saharan Africa, East Africa, Asia, and the Americas are infected with one or more helminths, making parasitic diseases very expensive for public health [68-70]. The drugs used until now to treat these diseases are far from ideal, and many of them were introduced decades ago [71, 72]. Most of these drugs are related to serious adverse effects, which supports the low adherence to treatment by patients. Moreover, these drugs have limited efficacy and can have their activity paralyzed due to the phenomenon of drug resistance $[72,73]$. The treatment of such parasitic diseases is mainly related to two factors: sanitation and health education, which when combined with the use of anthelmintic drugs promote the success of treatment and avoids occurrence of new cases [73, 74].

Ascariasis is a parasitic disease caused by infection with the nematode Ascaris lumbricoides, which is one of the soil-transmitted helminthiasis that head the list of the so-called "Neglected 
Diseases" [69, 75]. This parasitic disease has been associated with intestinal pathology, respiratory symptoms, and malnutrition in children from endemic areas [76, 77]. The indicated treatment for Ascariasis is based at anti-helminthic drugs against A. lumbricoides, which have been found to be safe and effective. Single oral doses of Albendazole, Mebendazole, and Pyrantel pamoate have demonstrated high cure rates against this parasite [69].

Albendazole and Mebendazole are Benzimidazole-based compounds, which are the most widely used anthelmintics until now [75]. These medicines are known as antimicrotubule agents due to their therapeutic effect by relating with the capacity of binding to free $\beta$-tubulin. This attachment inhibits tubulin polymerization and disrupts microtubules, which loses structure and function, leading the organism to death $[78,79]$. The selective toxicity of these agents results because Benzimidazoles bind parasite $\beta$-tubulin with much higher affinity than the mammalian protein [80]. However, there are evidences of drug resistance due to existence of cases where loss of efficacy has been reported after administration of a single dose [44, 81].

Schistosomiasis is also part of the list of "Neglected Diseases" and is responsible for infecting more than 200 million people in tropical and subtropical regions of the world, especially in sub-Saharan Africa [82, 83]. Introduced in the early 1980, Praziquantel (PZQ) is the drug of choice in the treatment of schistosomiasis. The detailed molecular mechanism of action of PZQ has not yet been fully explained, but it is related with tegumental damage and paralytic muscular contraction of parasites via influx of $\mathrm{Ca}^{2+}$ across the tegument $[74,84]$.

PZQ is a safe anthelmintic, but cases of drug resistance are already known, such as reported for ascariasis. The first field report came from a new and epidemic focus in northern Senegal [85]. Many infections that persisted after treatment and were not detected by the usual diagnostic methods (with eggs per gram below the detection limit of the coprological techniques) required repeated or very sensitive examinations [86, 87]. Thus, the recommended doses of medicine for schistosomiasis should be considered subcurative [88], and it is safe to assume that in schistosome populations, some individual parasites are tolerant to the drug, at least at the usual dosages [73].

The phenomenon of anthelmintic resistance is a genetic modification that can be defined as a state of insensitivity or decreased sensitivity to the effect of a determined drug concentration, conferring a number of parasites of a given population the capacity to survive the pharmacological effect of recommended therapeutic doses of an anthelmintic [75]. In veterinary practice, frequent treatment of closed populations has led to a serious problem of anthelmintic drug resistance, which is now largely irreversible [73]. Thus, it is urgently needed to search and develop sensitive tools for early detection and monitoring of drug resistance, as well as new anthelmintic evaluation of drug combinations for parasite control.

Drug discovery is an iterative process where different strategies can be applied. Usually, it can be divided into several distinct stages, starting with target identification and validation, proceeding to assay development and screening (whole cell or target molecular basis) and ending with hits identification. Thenceforward, it is necessary to synthesize and evaluate analogues to verify the structure-activity relationships and identify the elected hit; to optimize 
its structure, interactive medicinal chemistry is used, and finally, it is done on all preclinical development before the clinical evaluation [71].

Target-based drug discovery starts with the identification and elucidation of the function of a potential therapeutic drug target and understanding its role in the disease process. Drug targets are, basically, molecular structures that will undergo a specific interaction with chemicals (drugs), which are administered to treat or diagnose a disease. Most of the times, drug targets are enzymes or proteins involved in biological activity, which are produced by expression of active genes in a cell [70]. Furthermore, it is interesting to develop drugs whose targets include biochemical pathways, such as cellular physiological process or cell signalling molecules. For example, it has been reported as an inhibitor of programmed cell death pathway (apoptosis) to be a potential small molecule acting on a caspase to control schistosomiasis caused by S. japonicum [89]. Therefore, metabolic pathways can also be explored as therapeutic targets, where there should be a clear understanding of anthelmintics mechanisms of action operating in helminth parasites [70].

For exploratory studies as cited above, metabolomics is a very powerful tool to connect parasite metabolism and its interaction with the drug of choice. To better understand some of these mechanisms, chromatographic techniques combined with MS have been employed for chemical characterization of adult schistosomes and PZQ metabolites in the host [90, 91]. In a study from 2015, researchers used MS Imaging (MALDI-MSI) to better understand some of the mechanisms of interaction between PZQ and schistosomes. The researchers were able to characterize both sexes of $S$. mansoni adult worms (BH strain) treated with PZQ and identified the spatial distribution of chemical markers. In addition to tegumental damage, PZQ significantly changed the metabolic profile of both sexes of S. mansoni [49]. These data are supported by a recent work where tegumental damage of schistosomes under PZQ exposure was demonstrated through scanning electronic microscopy and optical microscopy [92]. These findings could be helpful for understanding possible targets and pathways of this antischistosomal drug and demonstrates the versatility and potential use of mass spectrometrybased metabolomics for identification of drug targets.

\section{Conclusions}

New tools are now available for biomedical research, and mass spectrometry techniques are among them. In "omics" research, huge opportunities are rising and enable to understand mechanistic basis for biological differences. For example, the absence of an enzyme in determined disease might be compared with healthy samples, unveiling significant molecular differences that clarify the mechanisms of action associated with a genetic failure. This new approach for biological systems allows the correlation between genotype and phenotype aspects, which is a complex process nowadays. Metabolomics studies are now giving the molecular profile of health or disease just like a "snapshot" of cell physiology. It is up to us scientists to establish the right and coherent correlations between metabolic patterns and biological alterations. 
Although helminthiasis still consists of a neglected disease, concerning all the discussed topics, it is possible to keep looking for new strategies that help to control and treat these infections. The groups of molecules identified by metabolomics approaches, exemplified as MS, might be tested and correlated with the proposed hypothesis, and then it may be possible to finally understand the parasites metabolism. In this way, it will be possible to comprehend the mechanisms of all different lifecycle stages, expanding the field of action for parasite control. In addition, metabolomics can bring light to the designing of new therapeutic drugs, highly effective and specific, reducing collateral effects and drug resistance.

Therefore, mass spectrometry and metabolomics show huge potential as new tools to unveil metabolism of different biological systems, including helminths. The more scientists unveil about metabolism of parasites and parasite-host interactions, the greater the chances of improving public health aiming a better future.

\section{Author details}

Estela O. Lima, Cibele Z. Esteves, Diogo N. Oliveira, Tatiane M. Guerreiro, Carlos F. O. R. Melo and Rodrigo R. Catharino*

*Address all correspondence to: rrcatharino@gmail.com Innovare Laboratory of Biomarkers, University of Campinas, Brazil

\section{References}

[1] Wilson DF. Regulation of cellular metabolism: programming and maintaining metabolic homeostasis. Journal of Applied Physiology (Bethesda, Md: 1985). 2013;115(11): 1583-8. Epub 2013/10/12.

[2] Doolittle WF. A paradigm gets shifty. Nature. 1998;392(6671):15-6.

[3] Cox FEG. History of human parasitology. Clinical Microbiology Reviews. 2002;15(4): 595-612.

[4] Hare DJ, New EJ. On the outside looking in: redefining the role of analytical chemistry in the biosciences. Chemical Communications. 2016;52(58):8918-34.

[5] Cox J, Mann M. Is proteomics the new genomics? Cell. 2007;130(3):395-8.

[6] Johnston DA, Blaxter ML, Degrave WM, Foster J, Ivens AC, Melville SE. Genomics and the biology of parasites. Bioessays. 1999;21(2):131-47.

[7] Wenk MR. Lipidomics of host-pathogen interactions. FEBS Letters. 2006;580(23): 5541-51. 
[8] Kafsack BF, Llinás M. Eating at the table of another: metabolomics of host-parasite interactions. Cell Host \& Microbe. 2010;7(2):90-9.

[9] Roessner U, Bowne J. What is metabolomics all about? Biotechniques. 2009;46(5):363.

[10] Weckwerth W. Metabolomics: an integral technique in systems biology. Bioanalysis. 2010;2(4):829-36.

[11] Patti GJ, Yanes O, Siuzdak G. Innovation: metabolomics: the apogee of the omics trilogy. Nature Reviews Molecular Cell Biology. 2012;13(4):263-9.

[12] Pan Z, Raftery D. Comparing and combining NMR spectroscopy and mass spectrometry in metabolomics. Analytical and Bioanalytical Chemistry. 2007;387(2):525-7.

[13] Katajamaa M, Orešič M. Data processing for mass spectrometry-based metabolomics. Journal of Chromatography A. 2007;1158(1):318-28.

[14] Harrigan GG, Goodacre R. Metabolic Profiling: Its Role in Biomarker Discovery and Gene Function Analysis. Springer Science \& Business Media; Dordrecht, Netherlands. 2012.

[15] Ellis DI, Goodacre R. Metabolic fingerprinting in disease diagnosis: biomedical applications of infrared and Raman spectroscopy. Analyst. 2006;131(8):875-85.

[16] Kell DB, Brown M, Davey HM, Dunn WB, Spasic I, Oliver SG. Metabolic footprinting and systems biology: the medium is the message. Nature Reviews Microbiology. 2005;3(7):557-65.

[17] Weljie AM, Newton J, Mercier P, Carlson E, Slupsky CM. Targeted profiling: quantitative analysis of $1 \mathrm{H}$ NMR metabolomics data. Analytical Chemistry. 2006;78(13):4430-42.

[18] Barding Jr GA, Salditos R, Larive CK. Quantitative NMR for bioanalysis and metabolomics. Analytical and Bioanalytical Chemistry. 2012;404(4):1165-79.

[19] Pettegrew JW. NMR: Principles and Applications to Biomedical Research. Springer Science \& Business Media; New York - USA. 2012.

[20] Han X, Gross RW. Structural determination of picomole amounts of phospholipids via electrospray ionization tandem mass spectrometry. Journal of the American Society for Mass Spectrometry. 1995;6(12):1202-10.

[21] Venter A, Nefliu M, Cooks RG. Ambient desorption ionization mass spectrometry. Trends in Analytical Chemistry. 2008;27(4):284-90.

[22] DeHaven CD, Evans AM, Dai H, Lawton KA. Organization of GC/MS and LC/MS metabolomics data into chemical libraries. Journal of Cheminformatics. 2010;2(1):9.

[23] Eppe G, Focant J-F, Pirard C, De Pauw E. PTV-LV-GC/MS/MS as screening and complementary method to HRMS for the monitoring of dioxin levels in food and feed. Talanta. 2004;63(5):1135-46. 
[24] Canuto GA, da Cruz PL, Faccio AT, Klassen A, Tavares MF. Neglected diseases prioritized in Brazil under the perspective of metabolomics: a review. Electrophoresis. 2015;36(18):2336-47.

[25] Holmes E. The evolution of metabolic profiling in parasitology. Parasitology. 2010;137(09):1437-49.

[26] Furlong S. Unique roles for lipids in Schistosoma mansoni. Parasitology Today. 1991;7(2): $59-62$.

[27] Ferreira MS, de Oliveira DN, de Oliveira RN, Allegretti SM, Catharino RR. Screening the life cycle of Schistosoma mansoni using high-resolution mass spectrometry. Analytica Chimica Acta. 2014;845:62-9.

[28] Retra K, Schmitz M, Yazdanbakhsh M, Tielens AG, Brouwers JF, van Hellemond JJ. The tegumental surface membranes of Schistosoma mansoni are enriched in parasite-specific phospholipid species. International Journal for Parasitology. 2015;45(9):629-36.

[29] Haslam SM, Morris HR, Dell A. Mass spectrometric strategies: providing structural clues for helminth glycoproteins. Trends in Parasitology. 2001;17(5):231-5.

[30] Smit CH, van Diepen A, Nguyen DL, Wuhrer M, Hoffmann KF, Deelder AM, et al. Glycomic analysis of life stages of the human parasite Schistosoma mansoni reveals developmental expression profiles of functional and antigenic glycan motifs. Molecular \& Cellular Proteomics. 2015;14(7):1750-69.

[31] Khoo K-H, Chatterjee D, Caulfield JP, Morris HR, Dell A. Structural mapping of the glycans from the egg glycoproteins of Schistosoma mansoni and Schistosoma japonicum: identification of novel core structures and terminal sequences. Glycobiology. 1997;7(5): 663-77.

[32] Yoshioka L, Zanotti-Magalhães EM, Magalhães LA, Linhares AX. Schistosoma mansoni: estudo da patogenia da linhagem Santa Rosa (Campinas, SP, Brasil) em camundongos. Revista da Sociedade Brasileira de Medicina Tropical. 2002;35(3):203-7.

[33] Jang-Lee J, Curwen RS, Ashton PD, Tissot B, Mathieson W, Panico M, et al. Glycomics analysis of Schistosoma mansoni egg and cercarial secretions. Molecular \& Cellular Proteomics. 2007;6(9):1485-99.

[34] Ferreira MS, Oliveira DN, Oliveira RN, Allegretti SM, Vercesi AE, Catharino RR. Mass spectrometry imaging: a new vision in differentiating Schistosoma mansoni strains. Journal of Mass Spectrometry. 2014;49(1):86-92.

[35] Meyer S, Van Liempt E, Imberty A, Van Kooyk Y, Geyer H, Geyer R, et al. DC-SIGN mediates binding of dendritic cells to authentic pseudo-LewisY glycolipids of Schistosoma mansoni cercariae, the first parasite-specific ligand of DC-SIGN. Journal of Biological Chemistry. 2005;280(45):37349-59.

[36] Wuhrer M, Dennis RD, Doenhoff MJ, Lochnit G, Geyer R. Schistosoma mansoni cercarial glycolipids are dominated by Lewis $\mathrm{X}$ and pseudo-Lewis $\mathrm{Y}$ structures. Glycobiology. 2000;10(1):89-101. 
[37] Van Hellemond JJ, Retra K, Brouwers JF, van Balkom BW, Yazdanbakhsh M, Shoemaker $\mathrm{CB}$, et al. Functions of the tegument of schistosomes: clues from the proteome and lipidome. International Journal for Parasitology. 2006;36(6):691-9.

[38] Pullan RL, Smith JL, Jasrasaria R, Brooker SJ. Global numbers of infection and disease burden of soil transmitted helminth infections in 2010. Parasites \& Vectors. 2014;7(1):1.

[39] Malaria GH-DoPDa. The Burden of Ascariasis 2011 Available from: http:// www.cdc.gov/globalhealth/ntd/diseases/ascaris_burden.html

[40] Committee WE. Prevention and Control of Schistosomiasis and Soil-Transmitted Helminthiasis. World Health Organization, Technical Report Series, No. 912; Geneva, Switzerland. 2002; vol. i-vi:1-57.

[41] Lo NC, Andrews JR, Bogoch II. Improving helminth treatment access: costs and opportunities. The Lancet Infectious Diseases. 2016;16(7):762-4.

[42] Organization WH. Investing to Overcome the Global Impact of Neglected Tropical Diseases: Third WHO Report on Neglected Diseases 2015. World Health Organization; Geneva, Switzerland. 2015.

[43] Shalaby HA. Anthelmintics resistance; how to overcome it? Iranian Journal of Parasitology. 2013;8(1):18.

[44] Albonico M, Engels D, Savioli L. Monitoring drug efficacy and early detection of drug resistance in human soil-transmitted nematodes: a pressing public health agenda for helminth control. International Journal for Parasitology. 2004;34(11):1205-10.

[45] Osei-Atweneboana MY, Eng JK, Boakye DA, Gyapong JO, Prichard RK. Prevalence and intensity of Onchocerca volvulus infection and efficacy of ivermectin in endemic communities in Ghana: a two-phase epidemiological study. The Lancet. 2007;369(9578): 2021-9.

[46] Araujo P. Observations on the early ecdyses of larvae of Ascaris lumbricoides, A. suum and Toxocara canis. Revista do Instituto de Medicina Tropical de São Paulo. 1972;14(2): 83-90.

[47] Artigas PdT, Ueta MT. About the evolution of Ascaris lumbricoides Linnaeus, 1758, in the larval stage inside the egg. Memories of Butantan Institute. 1989;51(1):15-24.

[48] Ligocka A, Paluszak Z. Effectiveness of different sanitization technologies on the inactivation of Ascaris suum eggs in organic waste. Bulletin of the Veterinary Institute in Pulawy. 2009;53:641-4.

[49] Ferreira MS, de Oliveira RN, de Oliveira DN, Esteves CZ, Allegretti SM, Catharino RR. Revealing praziquantel molecular targets using mass spectrometry imaging: an expeditious approach applied to Schistosoma mansoni. International Journal for Parasitology. 2015;45(6):385-91. 
[50] Keiser J, Duthaler U, Utzinger J. Update on the diagnosis and treatment of food-borne trematode infections. Current Opinion in Infectious Diseases. 2010;23(5):513-20.

[51] Lima EdO, de Macedo CS, Esteves CZ, de Oliveira DN, Pessolani MCV, Nery JAdC, et al. Skin imprinting in silica plates: a potential diagnostic methodology for leprosy using high-resolution mass spectrometry. Analytical Chemistry. 2015;87(7):3585-92.

[52] Melo CFOR, Esteves CZ, de Oliveira RN, Guerreiro TM, de Oliveira DN, de Oliveira Lima E, et al. Early developmental stages of Ascaris lumbricoides. Parasitology Research. 2016. DOI: 10.1007/s00436-016-5183-2; 1-8.

[53] Dhanasekaran DN, Reddy EP. JNK signaling in apoptosis. Oncogene. 2008;27(48): 6245-51.

[54] Kumar A, Byun H-S, Bittman R, Saba JD. The sphingolipid degradation product trans-2hexadecenal induces cytoskeletal reorganization and apoptosis in a JNK-dependent manner. Cellular Signalling. 2011;23(7):1144-52.

[55] Lin A, Dibling B. The true face of JNK activation in apoptosis. Aging Cell. 2002;1(2): $112-6$.

[56] Golden JW, Riddle DL. A pheromone influences larval development in the nematode Caenorhabditis elegans. Science. 1982;218(4572):578-80.

[57] Dold C, Holland CV. Ascaris and ascariasis. Microbes and Infection. 2011;13(7):632-7.

[58] Hadley NF. Surface waxes and integumentary permeability: lipids deposited on or associated with the surface of terrestrial plants and animals help protect them from a lethal rate of desiccation. American Scientist. 1980;68(5):546-53.

[59] Niwa R, Niwa YS. Enzymes for ecdysteroid biosynthesis: their biological functions in insects and beyond. Bioscience, Biotechnology, and Biochemistry. 2014;78(8):1283-92.

[60] Qu Z, Kenny NJ, Lam HM, Chan TF, Chu KH, Bendena WG, et al. How did arthropod sesquiterpenoids and ecdysteroids arise? Comparison of hormonal pathway genes in noninsect arthropod genomes. Genome Biology and Evolution. 2015;7(7):1951-9.

[61] Entchev EV, Kurzchalia TV. Requirement of sterols in the life cycle of the nematode Caenorhabditis elegans. Seminars in Cell \& Developmental Biology. 2005;16(2):175-182.

[62] Matyash V, Entchev EV, Mende F, Wilsch-Bräuninger M, Thiele C, Schmidt AW, et al. Sterol-derived hormone (s) controls entry into diapause in Caenorhabditis elegans by consecutive activation of DAF-12 and DAF-16. PLoS Biology. 2004;2(10):e280.

[63] Schlame M, Rua D, Greenberg ML. The biosynthesis and functional role of cardiolipin. Progress in Lipid Research. 2000;39(3):257-88.

[64] Takamiya S, Kita K, Wang H, Weinstein PP, Hiraishi A, Oya H, et al. Developmental changes in the respiratory chain of Ascaris mitochondria. Biochimica et Biophysica Acta (BBA)-Bioenergetics. 1993;1141(1):65-74. 
[65] Guan XL, Cestra G, Shui G, Kuhrs A, Schittenhelm RB, Hafen E, et al. Biochemical membrane lipidomics during Drosophila development. Developmental Cell. 2013;24(1):98-111.

[66] Carlson D, Langley P, Huyton P. Sex Pheromone of the Tsetse Fly: Isolation, Identification, and Synthesis of Contact Aphrodisiacs. American Association for the Advancement of Science. Washington DC - USA. 1978.

[67] Steiger S, Schmitt T, Schaefer HM. The origin and dynamic evolution of chemical information transfer. Proceedings of the Royal Society of London B: Biological Sciences. 2010:rspb20102285.

[68] Brooker S, Kabatereine NB, Smith JL, Mupfasoni D, Mwanje MT, Ndayishimiye O, et al. An updated atlas of human helminth infections: the example of East Africa. International Journal of Health Geographics. 2009;8(1):1.

[69] Hotez PJ, Brindley PJ, Bethony JM, King CH, Pearce EJ, Jacobson J. Helminth infections: the great neglected tropical diseases. The Journal of Clinical Investigation. 2008;118(4): 1311-21.

[70] Rana AK, Misra-Bhattacharya S. Current drug targets for helminthic diseases. Parasitology Research. 2013;112(5):1819-31.

[71] Pink R, Hudson A, Mouriès M-A, Bendig M. Opportunities and challenges in antiparasitic drug discovery. Nature Reviews Drug Discovery. 2005;4(9):727-40.

[72] Renslo AR, McKerrow JH. Drug discovery and development for neglected parasitic diseases. Nature Chemical Biology. 2006;2(12):701-10.

[73] Geerts S, Gryseels B. Drug resistance in human helminths: current situation and lessons from livestock. Clinical Microbiology Reviews. 2000;13(2):207-22.

[74] van den Enden E. Pharmacotherapy of helminth infection. Expert Opinion on Pharmacotherapy. 2009;10(3):435-51.

[75] Hagel I, Giusti T. Ascaris lumbricoides: an overview of therapeutic targets. Infectious Disorders-Drug Targets (Formerly Current Drug Targets-Infectious Disorders). 2010;10(5):349-67.

[76] Hagel I, Cabrera M, Hurtado M, Sanchez P, Puccio F, Di Prisco M, et al. Infection by Ascaris lumbricoides and bronchial hyper reactivity: an outstanding association in Venezuelan school children from endemic areas. Acta Tropica. 2007;103(3):231-41.

[77] Stephenson LS, Latham MC, Ottesen E. Malnutrition and parasitic helminth infections. Parasitology. 2000;121(S1):S23-S38.

[78] Lacey E. The role of the cytoskeletal protein, tubulin, in the mode of action and mechanism of drug resistance to benzimidazoles. International Journal for Parasitology. 1988;18(7):885-936. 
[79] Lacey E. Mode of action of benzimidazoles. Parasitology Today. 1990;6(4):112-5.

[80] Friedman PA, Platzer EG. Interaction of anthelmintic benzimidazoles and benzimidazole derivatives with bovine brain tubulin. Biochimica et Biophysica Acta (BBA)General Subjects. 1978;544(3):605-14.

[81] Keiser J, Utzinger J. Efficacy of current drugs against soil-transmitted helminth infections: systematic review and meta-analysis. Jama. 2008;299(16):1937-48.

[82] Colley DG, Bustinduy AL, Secor WE, King CH. Human schistosomiasis. The Lancet. 2014;383(9936):2253-64.

[83] Steinmann P, Keiser J, Bos R, Tanner M, Utzinger J. Schistosomiasis and water resources development: systematic review, meta-analysis, and estimates of people at risk. The Lancet Infectious Diseases. 2006;6(7):411-25.

[84] Bygott J, Chiodini P. Praziquantel: neglected drug? Ineffective treatment? Or therapeutic choice in cystic hydatid disease? Acta Tropica. 2009;111(2):95-101.

[85] Gryseels B, Stelma F, Talla I, Van Dam G, Polman K, Sow S, et al. Epidemiology, immunology and chemotherapy of Schistosoma mansoni infections in a recently exposed community in Senegal. Tropical and Geographical Medicine. 1993;46(4 Spec No):209-19.

[86] De Vlas S, Gryseels B. Underestimation of Schistosoma mansoni prevalences. Parasitology Today. 1992;8(8):274-7.

[87] Gryseels B, de Vlas SJ. Worm burdens in schistosome infections. Parasitology Today. 1996;12(3):115-9.

[88] Doenhoff MJ. Is schistosomicidal chemotherapy sub-curative? Implications for drug resistance. Parasitology Today. 1998;14(10):434-5.

[89] Peng J, Yang Y, Feng X, Cheng G, Lin J. Molecular characterizations of an inhibitor of apoptosis from Schistosoma japonicum. Parasitology Research. 2010;106(4):967-76.

[90] Meier H, Blaschke G. Investigation of praziquantel metabolism in isolated rat hepatocytes. Journal of Pharmaceutical and Biomedical Analysis. 2001;26(3):409-15.

[91] van Balkom BW, van Gestel RA, Brouwers JF, Krijgsveld J, Tielens AG, Heck AJ, et al. Mass spectrometric analysis of the Schistosoma mansoni tegumental sub-proteome. Journal of Proteome Research. 2005;4(3):958-66.

[92] Pinto-Almeida A, Mendes T, de Oliveira RN, Corrêa SdAP, Allegretti SM, Belo S, et al. Morphological characteristics of Schistosoma mansoni PZQ-resistant and-susceptible strains are different in presence of Praziquantel. Frontiers in Microbiology. 2016;7:11. 
